\title{
Trunk muscle fatigue during a lateral isometric hold test: what are we evaluating?
}

Isabelle Pagé ${ }^{1}$ and Martin Descarreaux ${ }^{2^{*}}$

\begin{abstract}
Background: Side bridge endurance protocols have been suggested to evaluate lateral trunk flexor and/or spine stabilizer muscles. To date, no study has investigated muscle recruitment and fatigability during these protocols. Therefore the purpose of our study was to quantify fatigue parameters in various trunk muscles during a modified side bridge endurance task (i.e. a lateral isometric hold test on a $45^{\circ}$ roman chair apparatus) and determine which primary trunk muscles get fatigued during this task. It was hypothesized that the ipsilateral external oblique and lumbar erector spinae muscles will exhibit the highest fatigue indices.

Methods: Twenty-two healthy subjects participated in this study. The experimental session included left and right lateral isometric hold tasks preceded and followed by 3 maximal voluntary contractions in the same position. Surface electromyography (EMG) recordings were obtained bilaterally from the external oblique, rectus abdominis, and L2 and L5 erector spinae. Statistical analysis were conducted to compare the right and left maximal voluntary contractions (MVC), surface EMG activities, right vs. left holding times and decay rate of the median frequency as the percent change from the initial value $\left(\mathrm{NMF}_{\text {slope }}\right)$.

Results: No significant left and right lateral isometric hold tests differences were observed neither for holding times (97.2 $\pm 21.5 \mathrm{sec}$ and $96.7 \pm 24.9 \mathrm{sec}$ respectively) nor for pre and post fatigue root mean square during MVCs. However, participants showed significant decreases of MVCs between pre and post fatigue measurements for both the left and right lateral isometric hold tests. Statistical analysis showed that a significantly $\mathrm{NMF}_{\text {slope }}$ of the ipsilateral external oblique during both conditions, and a $\mathrm{NMF}_{\text {slope }}$ of the contralateral $\mathrm{L} 5$ erector spinae during the left lateral isometric hold test were steeper than those of the other side's respective muscles. Although some participants presented positive $\mathrm{NMF}_{\text {slope }}$ for some muscles, each muscle presented a mean negative $\mathrm{NMF}_{\text {slope }}$ significantly different from 0.

Conclusions: Although the fatigue indices suggest that the ipsilateral external oblique and contralateral L 5 erector spinae show signs of muscle fatigue, this task seems to recruit a large group of trunk muscles. Clinicians should not view this task as evaluating specifically lateral trunk flexors, but rather as providing an indication of the general endurance and stabilisation capacity of the trunk.
\end{abstract}

Keywords: Side bridge test, Muscle fatigue, Trunk muscle, Functional testing

\section{Background}

Over the past two decades, lumbar spine stability has become an integral part of the low back pain assessment and treatment strategies, especially given its potential link to injury mechanisms and the ongoing clinical efforts directed toward enhancing stability in patients [1]. Furthermore, an increasing number of researchers and clinicians consider the strategy used by patients to

\footnotetext{
* Correspondence: martin.descarreaux@uqtr.ca

²Département de chiropratique, Université du Québec à Trois-Rivières, TroisRivières, G9A 5H7, Canada

Full list of author information is available at the end of the article
}

activate their abdominal muscles to be central to the stability theme. It has been demonstrated that bracing, defined as the increase of torso stiffness by the activation of all abdominal muscles and back extensors muscles, produces greater stability than hollowing, which consist of the activation of the transversus abdominis and internal oblique muscles in healthy subjects $[1,2]$. As a corollary, a variety of trunk coactivation exercise protocols are frequently used in daily clinical practice for low back pain prevention in healthy patients, rehabilitation in low back pain patients, or in order to evaluate trunk muscle function. The quadratus lumborum, external

\section{Biomed Central}


oblique, internal oblique, iliocostalis, longissimus and intertransversalis are believed to act as spine stabilizers when contracting bilaterally and as lateral trunk flexors by pulling the rib cage toward the hip when contracting unilaterally [3].

McGill et al. [4] suggested that a difference between left and right endurance time of the trunk flexors, extensors and lateral flexors muscles would predict who is at greater risk of back problem. As no exercise can evaluate all muscles involved in lumbar spine stability, evaluation in the 3 planes has to be done separately. Side bridge exercise protocols have been suggested to evaluate torso muscles in frontal plane. Such exercises are usually executed in a position where the participant lies down sideways with support of one arm and are named after the side of the arm support (e.g. left side bridge $=$ left arm support) [5] Variants to the protocol initially described by McGill have also been described [5-7].

A wide range of use of these exercises has been presented in the literature. Some authors proposed comparing muscle balance by evaluating holding times, whereas others assessed the use of maximal voluntary contraction or isometric contraction of short duration to evaluate muscle recruitment.

McGill et al. [8] evaluated the holding times of 75 healthy subjects (mean age of 23 years) during side bridge protocols and obtained mean times of $81 \pm 34 \mathrm{sec}$ and $85 \pm 36 \mathrm{sec}$ for right and left side bridge respectively. Other authors [9] reported similar mean times, i.e. $87.5 \pm 36.4 \mathrm{sec}$ and $92.0 \pm 45.8 \mathrm{sec}$ for right and left side bridges respectively, in a group of 24 healthy subjects (mean age of 35.3 years). McGill [10] has proposed that endurance scores during side bridges could be interpreted by using a right side to left side holding time ratio. A discrepancy of over 0.05 in the ratio would suggest unbalanced endurance. McGill et al. [8] also examined the intra-rater reliability of this test in 5 subjects on 5 consecutive days and at 8 weeks (follow-up) and got an excellent reliability coefficient of over .96 . Evans et al. [9] reported high intra-rater and inter-rater reliability with the lowest coefficient being .81 and .82 respectively.A number of studies have also provided information about muscle recruitment during side bridge tests. McGill et al. [11] reported ipsilateral (left side during side bridges with left supporting arm) trunk muscle activation of the quadratus lumborum, external oblique, rectus abdominis and lumbar erector spinae during isometric side bridges. These muscles presented 54\%, $40 \%, 22 \%$ and $24 \%$ of their maximal voluntary contraction activity (MVCA) respectively. However, the small sample (4 participants) does not allow for much generalization. Ekstrom et al. [12] evaluated muscle activation during 5-second hold side bridges and reported an activation of up to $72 \%$ of MVCA of the gluteus medius, $69 \%$ of the external oblique, between $34 \%$ and $42 \%$ of the longissimus thoracis, lumbar multifidus and rectus abdominis and less of $21 \%$ of the gluteus maximus and harmstring muscles. In 2008, Ekstrom et al. [13] used the same protocol to evaluate the longissimus thoracis and lumbar multifidus activation on both sides during side bridges. The authors reported greater activation of the ipsilateral longissimus thoracis than of the lumbar multifidus (48-49\% vs. $32-33 \%$ of MVCA respectively) and greater activation on the ipsilateral side than on the contralateral side $(48-49 \%$ vs $7-8 \%$ of MVCA for the longissimus thoracis and $32-33 \%$ vs $14 \%$ of MVCA for the lumbar multifidus). They also evaluated muscle activation during maximal resistance in side bridge without trunk support (legs fixed and trunk unsupported) and reported greater activation of the ipsilateral (left muscle when left side up) longissimus thoracis at L1 than of the lumbar multifidus $(54-58 \%$ and $38-39 \%$ of MVCA respectively). They demonstrated a greater activation on the ipsilateral side than on the contralateral one $54-58 \%$ vs. $7-9 \%$ of MVCA for longissimus thoracis and $38-39 \%$ vs. $12-13 \%$ of MVCA for lumbar multifidus). In light of these studies, the external oblique, gluteus medius and back extensor muscles on the ipsilateral side of the side bridge seem to be the muscles with the greatest levels of activation during this exercise.

Although muscle activation during side bridge endurance and trunk lateral flexion has been described in a few studies, muscle recruitment and fatigability protocols have not been extensively studied. Therefore the purpose of our study was to quantify fatigue parameters in various trunk muscles during a modified side bridge endurance test (i.e. a lateral isometric hold test on a $45^{\circ}$ roman chair apparatus). A second objective was to determine which primary trunk muscles are fatigued during this functional task. We hypothesized that the ipsilateral external oblique and erector spinae muscles would exhibit the highest fatigue indices during the modified side bridge protocol.

\section{Methods \\ Participants}

Twenty-two healthy subjects (11 men, 11 women; mean age \pm SD: $24.55 \pm 5.00$ ) participated in this study. All participants were volunteers and gave their informed, written consent according to the protocol approved by the Université du Québec à Trois-Rivières (Canada) Ethics Committee.

\section{Experimental protocol}

The one hour experimental session included maximal voluntary contractions and a trunk muscle endurance task in a lateral isometric hold position. Participants' height and weight were measured prior to the experimental task. 
The Baecke-f questionnaire was also completed by all participants in order to assess their physical activity levels (daily participation in sports and leisure activity) [14]. The experimental task was thoroughly explained and demonstrated by the experimenter before any data were recorded.

\section{Maximal voluntary contraction}

The endurance task was preceded and followed by three maximal voluntary contractions (MVC) in the same position, and participants were allowed a 2-minute rest period between the MVC pre-endurance and endurance task, and 15 minutes between post-endurance MVC and the other side pre-endurance MVC. MVC assessments, conducted 2 minutes prior to, and immediately after each endurance protocol, were performed against a force transducer that measured trunk muscle strength in the lateral position in accordance with the procedure presented by Ledoux et al. [7]. Participants were tested in the same position they held in the endurance tasks. Verbal encouragements were provided to maximize voluntary contraction. MVCs were performed against a fixed harness around the shoulders connected inline to a uni-axial force transducer on the floor (NTEP-87-057A3 class III, Artech, Riverside, CA, USA). For each MVC trial, force data were recorded at a sampling rate of $1,000 \mathrm{~Hz}$ and filtered digitally with a fifth-order Butterworth filter $(10-\mathrm{Hz}$ low-pass cutoff frequency). The higher force value obtained in 3 consecutive 10-s trials was used as the reference for MVC.

\section{Trunk muscle endurance task}

Participants were asked to perform sustained isometric contractions of the trunk muscles in a lateral position for both left and right sides named according to the side up (Figure 1). The endurance tasks (left and right) were counterbalanced across participants to control for sequence order effects. The protocol was explained and demonstrated before any experimental task was undertaken. During the left lateral isometric hold test, participants were positioned on the right side on a 45 degrees Roman chair. Ledoux et al. [7] suggested that this protocol could be used as an alternative to the side bridge test. When used to assess muscle fatigability, it yielded endurance time slightly longer than to those reported by McGill[10]. The test was developed as an alternative to the side bridge test to evaluate endurance time in older adults and adults with upper limb injuries (who could not attain support off the floor). This test also creates the possibility to assess maximal voluntary contraction in the same position. The trunk, from the anterior superior iliac spine and up, was unsupported. Arm support was allowed prior to the endurance task. On the researcher's cue, the participants removed their arms from the

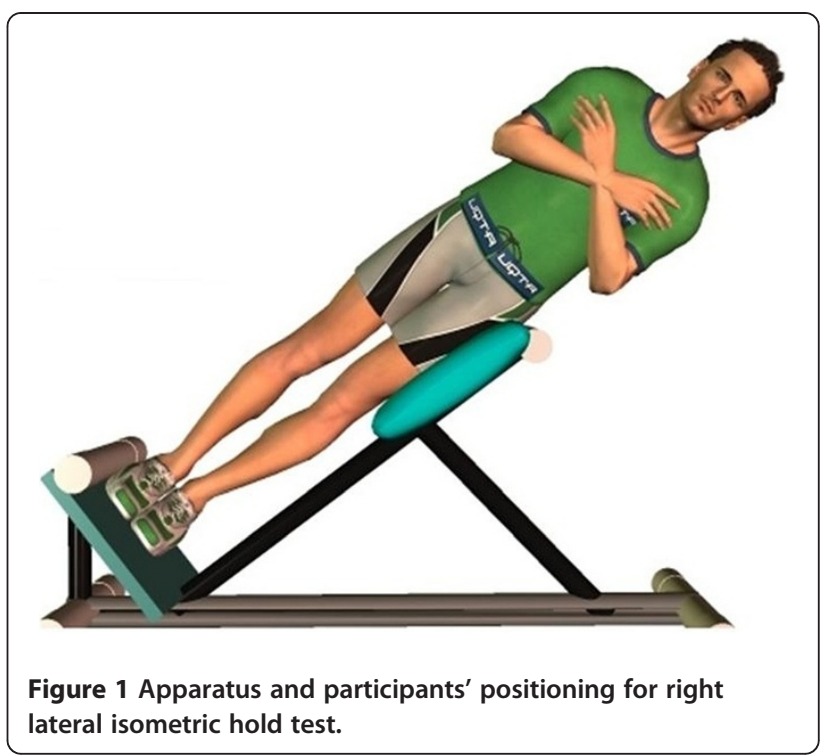

support and folded them across the chest with hands placed on the opposite shoulder. During the endurance task, participants were asked to keep their trunk and head in line with their lower limbs which were one above the other. The goal for all participants was to hold this position as long as they could. In order to ensure that participants abide by instructions, the same assistant observed the entire task for every participant and gave them verbal feedback to ensure proper position based on thorough observation. Failure to comply with instructions resulted in a warning by the assistant and the task was ended if the participant failed to follow instructions three times. Verbalized encouragement was provided throughout the test.

\section{Electromyography (EMG)}

Surface EMG data were collected with BiPole disposable surface $\mathrm{Ag}-\mathrm{AgCl}$ electrodes (Bortec biomedical, Calgary, Alberta, Canada) applied bilaterally on specific muscles in line with fiber direction. Inter-electrode distance was fixed at $2 \mathrm{~cm}$ and electrode diameter was $1 \mathrm{~cm}$. Muscle activity of the external oblique, rectus abdominis, lumbar erector spinae (L2 and L5 level) was recorded according to McGill et al. [15]. A ground electrode was placed on the left olecranon of each participant. Skin impedance was reduced by (1) shaving body hair, (2) gently abrading the skin with fine-grade sandpaper (Red Dot Trace Prep, $3 \mathrm{M}$, St. Paul, MN, USA), and (3) wiping the skin with alcohol swabs. EMG activity was recorded using a single differential Delsys Surface EMG sensor with a common mode rejection ratio of $92 \mathrm{~dB}$ at $60 \mathrm{~Hz}$, a noise level of $1.2 \mu \mathrm{V}$, a gain of $10 \mathrm{v} / \mathrm{v} \pm 1 \%$, an input impedance of $10^{15} \Omega$ (Model DE2.1, Delsys Inc., Boston, MA, USA) and sampled at $1,000 \mathrm{~Hz}$ with a 12 -bit $\mathrm{A} / \mathrm{D}$ converter (PCI 6024E, National Instruments, Austin, TX, USA). 
The EMG data were filtered digitally by a $10-$ to $450-\mathrm{Hz}$ band-pass, zero-lag, fifth-order Butterworth filter. They were collected by LabView (National Instruments) and processed by Matlab (MathWorks, Natick, MA, USA).

\section{Data analysis}

Maximal voluntary contraction (Newton) and maximal EMG root mean square values (RMS) (for each muscle) were obtained for every MVC trial to assess muscle fatigue. Muscular fatigue was assessed during the fatigue protocol through power spectral analysis. Median power frequency (MedF) was calculated from successive nonoverlapping windows of 250 ms by Fast-Fourier transformation. Least square linear regression analysis was applied to MedF time series (MedF as a function of time) to estimate the rate of decline $\left(\mathrm{MedF}_{\text {slope }}\right)$. In order to express the decay rate of MedF as the percent change from the initial value $\left(\mathrm{NMF}_{\text {slope }}\right.$ in $\left.\% \mathrm{~s}^{-1}\right), \mathrm{MedF}_{\text {slope }}$ were divided by the initial MedF $[16,17]$. The equation below was used to obtained individual holding time ratios:

\section{$\mid 1$ - (rightholdingtime/leftholdingtime) $\mid$}

\section{Statistical analysis}

Right and left maximal voluntary contractions (Newtons) and RMS were compared between pre and post endurance tasks with 2-tailed $t$-tests for dependent samples. 2-tailed $t$-tests for dependant samples were also used to compare holding times between left and right lateral isometric hold tests. In order to test for our main hypothesis, the $\mathrm{NMF}_{\text {slope }}$ of antagonist muscles in both conditions (e.g. left external oblique vs. right external oblique during left lateral isometric hold test) were compared using 2-tailed $t$-tests for independent samples. $T$-tests for independent samples (1-tailed) comparing $\mathrm{NMF}_{\text {slope }}$ to 0 were conducted for every muscle in both conditions to determine if statistically significant decay of NMF were induced by the fatigue protocols. Simple correlation tests were performed separately for each baseline characteristic and holding times in order to evaluated the possible correlations. Statistical significance was set at $p$ $<0.05$ for all analyses.

\section{Results}

\section{Participants' characteristics}

Table 1 reports the mean (SD) baseline characteristics of participants (means age, weight, height, body mass index, Baecke-f questionnaire score).

\section{Holding times and maximal voluntary contractions}

$T$-tests for dependant samples revealed no significant difference between sides for holding times $(p>0.88)$ but showed significant decreases of MVCs between pre and post endurance task measurements for both the left and right lateral isometric hold tests $(p<0.01$ and $p=0.02$ respectively). No significant differences were observed between RMS pre and post endurance task values $(p>0.05)$. The calculated mean individual holding time ratio showed a $0.119 \pm 0.118$ discrepancy between the right and left sides. Simple correlation tests revealed no significant correlation between holding times and each baseline characteristic $(p>0.05)$. Figure 2 and Table 2 present the mean \pm standard deviation for holding times and MVC statistical analysis.

\section{Muscles recruitment during endurance task and muscle fatigue indices}

Figure 3 presents the $\mathrm{NMF}_{\text {slope }}$ of external oblique and L5 erector spinae muscles for both sides during left and right lateral isometric hold tests. $T$-tests for independent samples comparing $\mathrm{NMF}_{\text {slope }}$ of antagonist muscles in both conditions (e.g. left external oblique vs. right external oblique during left lateral isometric hold test) showed a significantly greater decay rate of the $\mathrm{NMF}_{\text {slope }}$ of ipsilateral external oblique (i.e. left external oblique while left lateral isometric hold test comparing to right external oblique) during both conditions and of right L5 erector spinae during left lateral isometric hold test comparing to the left muscle. During right lateral isometric hold test, the left L5 erector spinae presented a trend toward a greater decay rate of the $\mathrm{NMF}_{\text {slope }}$ than the right one, but these differences were not significant $(p=0.08)$. No difference was observed in the other left versus right muscle comparisons (L2 erector spinae and rectus abdominis) in any of the conditions $(p>0.05)$. Table 3 presents results of $t$-tests for independent samples for $\mathrm{NMF}_{\text {slope }}$ of each muscle recorded during both conditions. Although some participants presented positive $\mathrm{NMF}_{\text {slope }}$ for some muscles, each muscle presented a mean negative $\mathrm{NMF}_{\text {slope }}$ significantly different from 0 at $p<0.05$.

\section{Discussion}

This study's main objectives were to quantify fatigue parameters in various trunk muscles during a lateral isometric hold test in healthy participants and to determine which primary trunk muscles are fatigued during this functional task. The ipsilateral external oblique, during

Table 1 Participants' characteristics (mean \pm SD)

\begin{tabular}{lr}
\hline & Mean \pm SD \\
\hline Age (years) & $24.55 \pm 5.00$ \\
Weight $(\mathrm{kg})$ & $70.62 \pm 8.86$ \\
Height $(\mathrm{m})$ & $1.72 \pm 0.09$ \\
Body mass index $\left(\mathrm{kg} / \mathrm{m}^{2}\right)$ & $23.94 \pm 2.78$ \\
Sport activity indices in Baecke-f questionnaire & $5.40 \pm 1.51$ \\
Free-time activity indices in Baecke-f questionnaire & $3.06 \pm 0.79$ \\
\hline
\end{tabular}




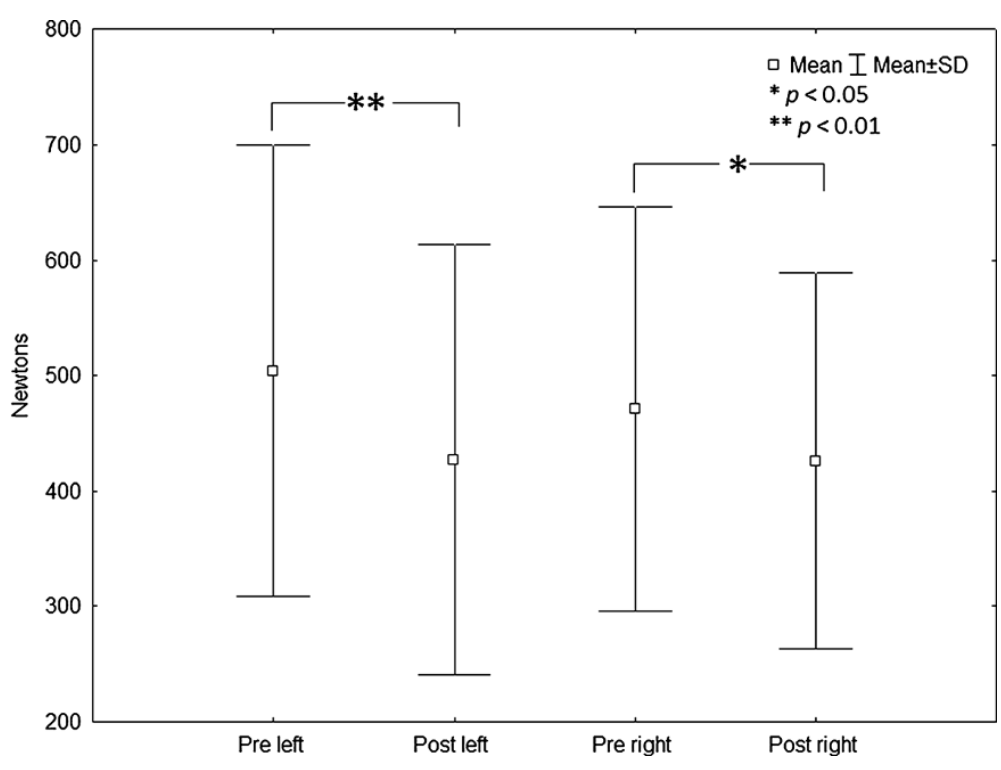

Figure $\mathbf{2}$ Left and right lateral isometric hold test MVCs before and after the endurance task.

Table 2 Left and right lateral isometric hold tests holding times and ratios (mean \pm SD)

\begin{tabular}{lc}
\hline & Mean \pm SD \\
\hline Left lateral isometric hold test holding times (sec) & $96.7 \pm 24.9$ \\
Right lateral isometric hold test holding times (sec) & $97.2 \pm 21.5$ \\
Holding time ratio & $0.119 \pm 0.118$ \\
$P$ value (between left and right lateral isometric hold tests) & 0.88
\end{tabular}

both lateral isometric hold tests, and the contralateral L5 erector spinae, during the left lateral isometric hold test, showed significantly steeper negative $\mathrm{NMF}_{\text {slope }}$ than the other side's respective muscles. The results also showed that all recorded trunk muscles presented fatigue parameters during lateral isometric hold tests on both sides. Thereby, these results provide a preliminary understanding of the trunk muscles tested during a lateral isometric hold task.

Even if the results showed a $\mathrm{NMF}_{\text {slope }}$ significantly different from 0 for the 8 muscles recorded, the percentage of decay did not exceed $0.07 \% \mathrm{~s}^{-1}$ which may raise questions with regard to the level of fatigue generated in individual muscles during the lateral hold. Despite the lack of normative data for fatigue parameters, other studies have reported steeper negative $\mathrm{NMF}_{\text {slope }}$ during trunk endurance protocols. Plamondon et al. [18] reported L3 erector spinae $\mathrm{NMF}_{\text {slope }}\left(\% \mathrm{~s}^{-1} \pm \mathrm{SD}\right)$ of $0.10 \pm 0.07$ and $0.13 \pm 0.05$ for women and men respectively during modified Sorenson intermittent contraction tasks. Mannion et al. [16,17] reported $\mathrm{NMF}_{\text {slope }}$ values up to $0.46 \pm 0.19$ during modified Biering-Sorenson test. The results of the present study could suggest that muscles not recorded in our experiment could act as primary contributors during the lateral isometric hold task. In fact, the quadratus lumborum, which activity can be predicted with surface EMG placed on L3 erector spinae [15], has been reported to act as a trunk lateral flexor when contracted unilaterally $[3,19]$, and some studies reported high levels of activity in this muscle during side bridges $[11,20]$. Lower limb muscle activity was not recorded in the present study, but gluteus medius, gluteus maximus and hamstring activation during different side bridges exercises has also been reported $[6,12,13]$.

As often seen in motor tasks, there are certainly multiple muscle recruitment strategies (redundancy) that can be selected by the central nervous system to optimize task performance during a lateral isometric hold test [21]. During this task, more than one muscle contribute to the torque generated, and a changing combination of muscle forces to maintain the isometric contraction may be an efficient strategy to improve performance. Motor variability has been shown to be an efficient strategy to reduce the development of muscle fatigue [22]. Our results suggest that a variable trunk co-contraction strategy where numerous muscles contribute to the generation of isometric force is selected during a lateral isometric hold test. A few authors also reported global activation of trunk muscles instead of specific muscle recruitment during trunk endurance task. Page et al. [23] reported fatigue of various trunk muscles (i.e. abdominal muscles, lumbar erector spinae, biceps femoris) during sustained isometric contractions of abdominal muscles whereas Plamondon et al. [18] reported 


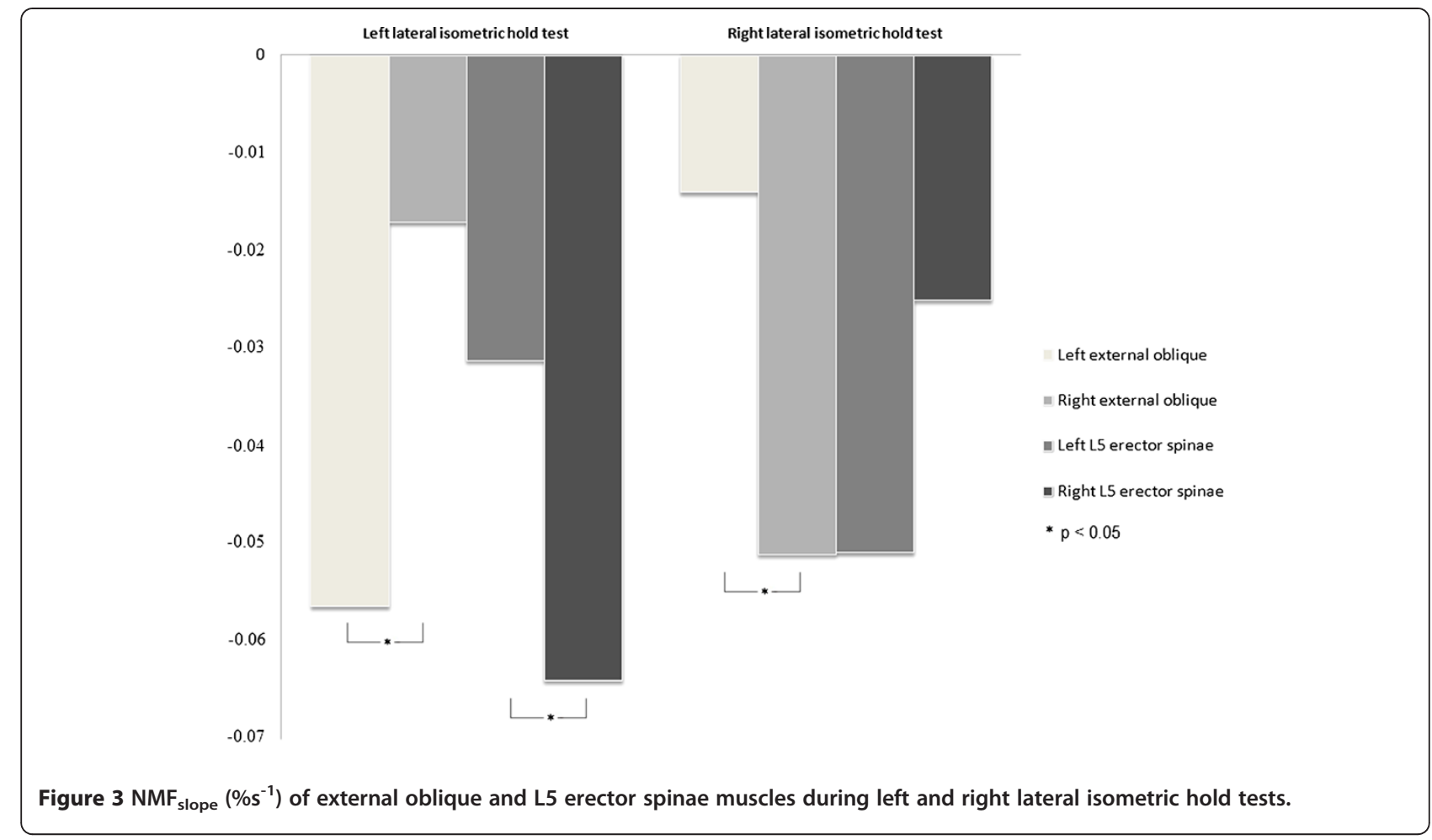

Table $3 \mathrm{NMF}_{\text {slope }} \boldsymbol{t}$-tests for left and right lateral isometric hold tests (mean \pm SD)

\begin{tabular}{|c|c|c|c|c|c|c|}
\hline \multirow[t]{2}{*}{ Muscles } & \multicolumn{3}{|c|}{ Left lateral isometric hold test } & \multicolumn{3}{|c|}{ Right lateral isometric hold test } \\
\hline & df & $\begin{array}{c}\mathrm{NMF}_{\text {slope }} \\
\left(\% \mathrm{~s}^{-1}\right)\end{array}$ & $\begin{array}{c}P \\
\text { values }\end{array}$ & df & $\begin{array}{c}\mathrm{NMF}_{\text {slope }} \\
\left(\% \mathrm{~s}^{-1}\right)\end{array}$ & $\begin{array}{c}P \\
\text { values }\end{array}$ \\
\hline Left rectus abdominis & 19 & $-0.025 \pm 0.054$ & 0.018 & 20 & $-0.037 \pm 0.043$ & $<0.001$ \\
\hline Right rectus abdominis & 18 & $-0.032 \pm 0.038$ & $<0.001$ & 20 & $-0.026 \pm 0.023$ & $<0.001$ \\
\hline Left external oblique & 18 & $-0.056 \pm 0.037$ & $<0.001$ & 20 & $-0.014 \pm 0.026$ & 0.0106 \\
\hline Right external oblique & 19 & $-0.017 \pm 0.030$ & 0.009 & 20 & $-0.051 \pm 0.039$ & $<0.001$ \\
\hline Left L2 erector spinae & 20 & $-0.023 \pm 0.031$ & $<0.001$ & 20 & $-0.034 \pm 0.054$ & 0.0041 \\
\hline Right L2 erector spinae & 20 & $-0.043 \pm 0.051$ & $<0.001$ & 20 & $-0.025 \pm 0.029$ & $<0.001$ \\
\hline Left $L 5$ erector spinae & 20 & $-0.031 \pm 0.053$ & 0.0011 & 17 & $-0.051 \pm 0.031$ & $<0.001$ \\
\hline Right L5 erector spinae & 18 & $-0.064 \pm 0.048$ & $<0.001$ & 20 & $-0.025 \pm 0.055$ & 0.022 \\
\hline
\end{tabular}

fatigue of lumbar erector spinae and hip extensor muscles (hamstring and gluteus maximus) during an intermittent modified Sorenson contraction task. Furthermore, other studies have reported the recruitment of several trunk muscles (e.g. quadratus lumborum, external oblique, rectus abdominis, lumbar and thoracis erector spinae, gluteus medius, lumbar multifidus, gluteus maximus and hamstrings) during side bridge tasks [11-13].

Our participants showed mean holding times similar to the ones reported in previous studies where lateral flexors endurance was evaluated using the side bridge position $[8,9]$. However, although only healthy participants were included in the study, a mean difference of more than $5 \%$ between both side holding times was observed. According to McGill, such differences in holding times during the side bridge position would suggest trunk muscle imbalance [10]. The relatively high variability of the right on left holding time ratio (i.e. a standard deviation of $11.8 \%$ ) suggests a wide variation of this ratio in healthy populations. Differences in testing protocols (isometric lateral hold versus side bridge) may explain these differences. However, one might question the clinical value of the proposed criteria suggesting that differences of more than $5 \%$ between right and left holding times characterize individuals with a history of 
disabling back troubles or an increased risk of back trouble [10]. Others studies are necessary to validate the use of a holding time ratio derived from the lateral isometric hold test as a normative data to evaluate muscle balance and function.

A few limitations need to be considered when interpreting the present results. For this study, only healthy young adults were recruited, and consequently generalization to other healthy or clinical populations may be limited. Only eight trunk muscles (surface EMG) were recorded, and other studies are necessary to evaluate the possible contribution and fatigue of several other trunk or lower limb muscles that could be recruited during lateral isometric hold task.

\section{Conclusions}

This study was conducted in order to identify muscles evaluated during a lateral isometric hold task. Although the fatigue indices suggest that ipsilateral external oblique and contralateral L5 erector spinae are significant contributors, all recorded muscles were active during the lateral isometric hold task. The lateral isometric hold, while different from the side bridge assessment for endurance because it does not involve support of the floor, seems to be adequate for the evaluation of lateral trunk flexors. Studies involving individuals with low back pain are needed to evaluate the clinical relevance of this procedure.

\section{Competing interests}

The author(s) declare that they have no competing interests.

\section{Acknowledgements}

The study was funded by the Chaire de Recherche en Chiropratique FRCQ, the Fonds de Recherche en Santé du Québec (FRSQ), the Institut de Recherche Robert-Sauvé en Santé et en Sécurité du Travail (IRSST) and the Canadian Chiropractic Association. Other contributors: Marie-Pierre Harvey and Maude Carroll who contributed to the experimentation.

\section{Author details}

'Département des sciences de l'activité physique, Université du Québec à Trois-Rivières, Trois-Rivières, G9A 5H7, Canada. ${ }^{2}$ Département de chiropratique, Université du Québec à Trois-Rivières, Trois-Rivières, G9A 5H7, Canada.

\section{Authors' contributions}

IP participated in the study design, experimentation and manuscript writing. MD participated in study design, data analysis, manuscript writing and revision. All authors read and approved the final manuscript.

Received: 21 November 2011 Accepted: 19 April 2012

Published: 19 April 2012

\section{References}

1. Grenier SG, McGill SM: Quantification of lumbar stability by using 2 different abdominal activation strategies. Arch Phys Med Rehabil 2007, 88 (1):54-62.

2. Richardson CA, Snijders CJ, Hides JA, Damen L, Pas MS, Storm J: The relation between the transversus abdominis muscles, sacroiliac joint mechanics, and low back pain. Spine (Phila Pa 1976) 2002, 27(4):399-405.

3. Wiatt E, Flanagan SP: Lateral trunk flexors and low back pain: endurance and bilateral asymmetry. Ath/10etic Therapy Today 2009, 14(3):10-12.
4. McGill SM, Grenier S, Kavcic N, Cholewicki J: Coordination of muscle activity to assure stability of the lumbar spine. J Electromyogr Kinesiol 2003, 13(4):353-359.

5. McGill SM: Low back exercises: evidence for improving exercise regimens. Phys Ther 1998, 78(7):754-765.

6. Konrad P, Schmitz K, Denner A: Neuromuscular evaluation of trunk-training exercises. J Athl Train 2001, 36(2):109-118.

7. Ledoux E, Dubois JD, Descarreaux M: Physical and psychosocial predictors of functional trunk capacity in older adults with and without low back pain. JMPT 2012 (In press).

8. McGill SM, Childs A, Liebenson C: Endurance times for low back stabilization exercises: clinical targets for testing and training from a normal database. Arch Phys Med Rehabil 1999, 80(8):941-944.

9. Evans K, Refshauge KM, Adams R: Trunk muscle endurance tests: reliability, and gender differences in athletes. J Sci Med Sport 2007, 10(6):447-455.

10. McGill S: Low back disorders: evidence-based prevention and rehabilitation. Champaign, IL: Human Kinetics; 2002.

11. McGill S, Juker D, Kropf P: Quantitative intramuscular myoelectric activity of quadratus lumborum during a wide variety of tasks. Clin Biomech (Bristol, Avon) 1996, 11(3):170-172.

12. Ekstrom RA, Donatelli RA, Carp KC: Electromyographic analysis of core trunk, hip, and thigh muscles during 9 rehabilitation exercises. J Orthop Sports Phys Ther 2007, 37(12):754-762.

13. Ekstrom RA, Osborn RW, Hauer PL: Surface electromyographic analysis of the low back muscles during rehabilitation exercises. $J$ Orthop Sports Phys Ther 2008, 38(12):736-745.

14. Bigard AX, Duforez F, Portero P, Guezennec CY: Détermination de l'activité physique par questionnaire: validation du questionnaire autoadministrable de Baecke. Sciences and Sports 1992, 7:215-221.

15. McGill $S$, Juker D, Kropf P: Appropriately placed surface EMG electrodes reflect deep muscle activity (psoas, quadratus lumborum, abdominal wall) in the lumbar spine. J Biomech 1996, 29(11):1503-1507.

16. Mannion AF, Connolly B, Wood K, Dolan P: The use of surface EMG power spectral analysis in the evaluation of back muscle function. $J$ Rehabil Res Dev 1997, 34(4):427-439.

17. Mannion AF, Dolan P: Electromyographic median frequency changes during isometric contraction of the back extensors to fatigue. Spine (Phila Pa 1976) 1994, 19(11):1223-1229.

18. Plamondon A, Trimble K, Lariviere C, Desjardins P: Back muscle fatigue during intermittent prone back extension exercise. Scand J Med Sci Sports 2004, 14(4):221-230.

19. Huang QM, Andersson $E$, Thorstensson A: Intramuscular myoelectric activity and selective coactivation of trunk muscles during lateral flexion with and without load. Spine (Phila Pa 1976) 2001, 26(13):1465-1472.

20. Kavcic N, Grenier S, McGill SM: Determining the stabilizing role of individual torso muscles during rehabilitation exercises. Spine (Phila Pa 1976) 2004, 29(11):1254-1265.

21. Latash ML, Scholz JP, Schoner G: Toward a new theory of motor synergies. Mot Control 2007, 11(3):276-308.

22. Madeleine P: On functional motor adaptations: from the quantification of motor strategies to the prevention of musculoskeletal disorders in the neck-shoulder region. Acta Physiol (Oxf) 2010, 199(Suppl 679):1-46.

23. Page I, Dubois JD, Descarreaux M: A comparison of 2 assessment protocols to specifically target abdominal muscle endurance. J Manipulative Physiol Ther 2011, 34(3):188-194.

doi:10.1186/2045-709X-20-12

Cite this article as: Pagé and Descarreaux: Trunk muscle fatigue during a lateral isometric hold test: what are we evaluating? Chiropractic \& Manual Therapies 2012 20:12. 